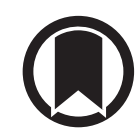

CrossMark

\title{
Pulmonary hypertension associated with left heart disease: efforts to improve the meaning of haemodynamic phenotypes
}

\author{
To the Editor:
}

We read with great interest the recent article by GERGES et al. [1] on partitioning pulmonary vascular resistance (PVR) at baseline and after inhaled nitric oxide (iNO) in patients with pulmonary hypertension associated with left heart disease (PH-LHD). The study highlighted that the increase of right ventricular (RV) afterload in isolated post-capillary $\mathrm{PH}$ (Ipc-PH) primarily depended on a passive backward transmission of left ventricular filling pressure and left atrial (LA) function, explaining the very high upstream resistance (Rup). Further afterload increase secondary to an elevated vessel resistance in combined post- and pre-capillary pulmonary hypertension $(\mathrm{Cpc}-\mathrm{PH})$, was associated with a lower Rup similar to that seen in idiopathic pulmonary arterial hypertension (iPAH) patients.

Pulmonary vascular disease (PVD) in PH-LHD in patients with heart failure is associated with a prevalence of pulmonary veins, capillaries and distal muscular pulmonary arteries (PAs) remodelling [2, 3]. Current dogma suggests that decreased pulmonary arterial capacitance (PAC) in pulmonary hypertension $(\mathrm{PH})$ is a consequence of distal proliferative PVD. We have shown that Ipc-PH patients with PVR and diastolic pulmonary gradient (DPG) within normal limits have a significant increase in area wall thickness and stiffening of proximal PA, and impairment of RV-to-pulmonary arterial coupling, suggesting the presence of early PVD and questioning the definition of "passive" PH-LHD [4]. We speculate that the presence of proximal PA wall disease in addition to the passive upstream transmission of elevated LA pressure may explain the significant lower PAC in Ipc-PH patients. The elevation of PVR and DPG would occur later with further reductions in PAC associated with distal PVD, as seen in Cpc-PH patients [4].

The definition of PH-LHD haemodynamic phenotypes is a matter of debate. The Ipc- $\mathrm{PH}$ and $\mathrm{Cpc}-\mathrm{PH}$ phenotypes defined in the most recent 2015 ESC/ERS guidelines [5] have been challenged due to their controversial prognostic role and the ambiguous classification of a significant proportion of patients [6]. Both the stationary (PVR) and the pulsatile (PAC) components of afterload exhibit an inverse hyperbolic relationship. The product of resistance and compliance (RC-time) is mostly constant in both healthy and diseased states, with the exception of a few clinical scenarios like elevated left-sided filling pressures, proximal chronic thromboembolic $\mathrm{PH}(\mathrm{CTEPH})$ and heart rate (HR) increase. In all these cases, PAC decreases proportionally more than the increase in PVR, RC-time decreases and the $\mathrm{R}$-C curve shifts downwards left $[7,8]$.

We have previously proposed an original dimensionless haemodynamic index, upstream impedance ( $\left.Z_{\text {up }}\right)$ ((TPG-DPG)/TPG) (where TPG is transpulmonary gradient), that enables the characterisation of the broad spectrum of dynamic afterload and predicts early outcome after pulmonary endarterectomy for CTEPH [9]. Replacing mean pulmonary arterial pressure (mPAP) with (sPAP+2dPAP)/3 (where sPAP and $\mathrm{dPAP}$ are systolic and diastolic PAP, respectively) in the numerator and with $\mathrm{PVR} \times \mathrm{SV} \times \mathrm{HR}+\mathrm{PAOP}$ (where SV is systolic volume and PAOP is pulmonary arterial occlusion pressure, end-expiratory automated digital mean measurements across the cardiac cycle) in the denominator, Zup is inversely

@ERSpublications

Upstream impedance could characterise the RV afterload and the relative contribution of large and small vessel disease in $\mathrm{PH}$, regardless of the PAOP, including the inverse relationship with $\mathrm{HR}$ as an indirect RV functional response http://ow.ly/fqvN30nbNFo

Cite this article as: Grignola JC, Trujillo P, Domingo E. Pulmonary hypertension associated with left heart disease: efforts to improve the meaning of haemodynamic phenotypes. Eur Respir J 2019; 53: 1801894 [https://doi.org/10.1183/13993003.01894-2018]. 


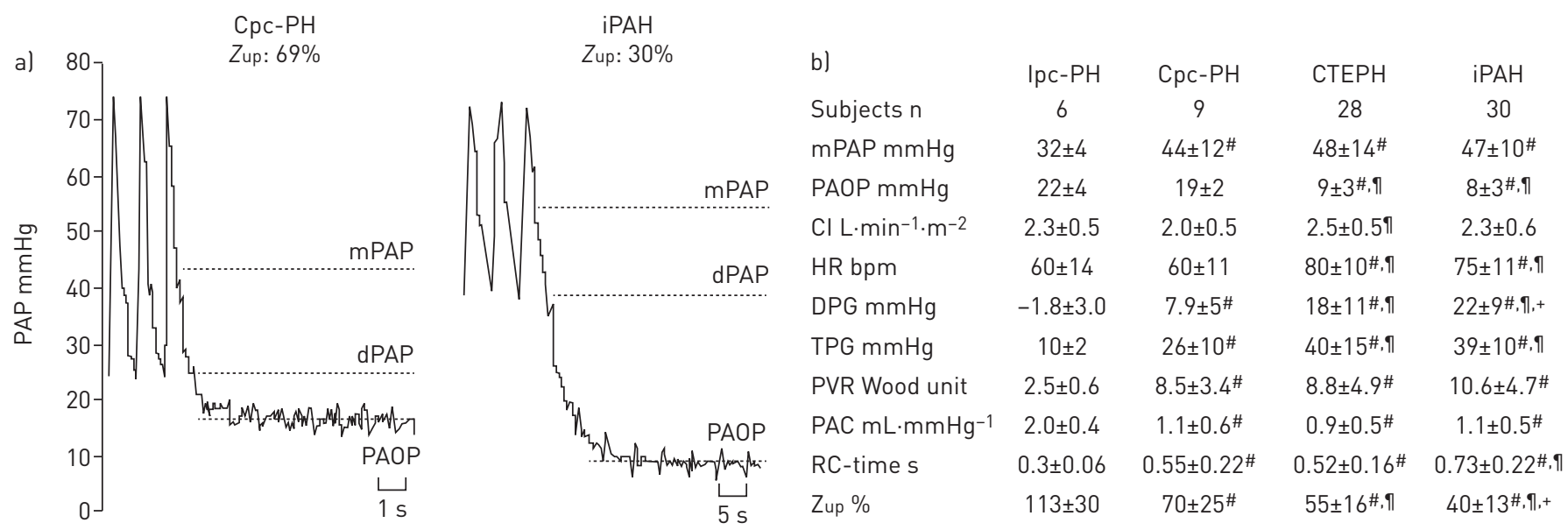

FIGURE 1 a) Pulmonary arterial occlusion pressure (PAOP) decay curve in a combined pre- and post-capillary pulmonary hypertension (Cpc-PH) patient and an idiopathic pulmonary arterial hypertension (iPAH) patient. b) Haemodynamic data. Data are presented as mean \pm SD, unless otherwise stated. PAP: pulmonary arterial pressure; Ipc-PH: isolated post-capillary pulmonary hypertension; CTEPH: chronic thromboembolic pulmonary hypertension; mPAP: mean PAP; dPAP: diastolic PAP; $\mathrm{Cl}$ : cardiac index; HR: heart rate; DPG: diastolic pulmonary gradient; TPG: transpulmonary gradient; PVR: pulmonary vascular resistance; PAC: total pulmonary arterial capacitance; RC-time: product of PVR $\times$ PAC; Zup: upstream impedance. ${ }^{\#}$ : $\mathrm{p}<0.05$ versus $\mathrm{Ipc}-\mathrm{PH}_{;}{ }^{\uparrow}: \mathrm{p}<0.05$ versus $\mathrm{Cpc}-\mathrm{PH}_{;}{ }^{+}: \mathrm{p}<0.05$ versus CTEPH.

related to PAC, PVR, and HR:

$$
\begin{aligned}
\mathrm{Zup} & =\frac{\mathrm{mPAP}-\mathrm{dPAP}}{\mathrm{mPAP}-\mathrm{PAOP}}=\frac{((\mathrm{sPAP}+2 \mathrm{dPAP}) / 3)-\mathrm{dPAP}}{\mathrm{PVR} \times \mathrm{SV} \times \mathrm{HR}}=\frac{\mathrm{sPAP}-\mathrm{dPAP} / 3}{\mathrm{PVR} \times \mathrm{SV} \times \mathrm{HR}} \\
& =\frac{\mathrm{SV} / 3 \mathrm{PAC}}{\mathrm{PVR} \times \mathrm{SV} \times \mathrm{HR}}=\frac{1}{3 \times \mathrm{PVR} \times \mathrm{PAC} \times \mathrm{HR}}
\end{aligned}
$$

In $\mathrm{PH}$ states, the greater increase of pulsatile to the stationary component of RV afterload determines elevated PA pressures with a large pulse pressure or "ventricularisation" and higher Zup values [10]. We hypothesised that $Z$ up could characterise the relative contribution of large and small vessels disease through its differential impact on the afterload components in $\mathrm{PH}$ states, regardless of the PAOP. We compare $Z$ up values in patients with PH-LHD (post-capillary PH), and operable CTEPH and iPAH (pre-capillary $\mathrm{PH}$ ).

We can see that $Z$ up values are higher in post-capillary $\mathrm{PH}$ than pre-capillary $\mathrm{PH}$, which is probably due to the greater pulsatile afterload associated with higher PAOP and lower HR. In pre-capillary $\mathrm{PH}$, the more proximal occlusive site causes a higher PA stiffness and an earlier and greater wave reflection, increasing total PVR but with a lower dPAP and PAC and a higher Zup in operable CTEPH than in iPAH. Similarly, among post-capillary $\mathrm{PH}$, the presence of proximal PVD determines the PAC decrease and the highest Zup in Ipc-PH patients. The elevation of PVR and DPG with more reductions in PAC associated with distal PVD, would explain the lower $Z$ up of $\mathrm{Cpc}-\mathrm{PH}$ than Ipc-PH. Despite the fact that the PA wall stiffness and the haemodynamic profile of $\mathrm{Cpc}-\mathrm{PH}$ patients are very similar compared to historical patients with PAH, Zup is significantly higher, which is likely to be due to lower HR and the post-capillary condition (figure 1) $[4,11]$.

We observed substantial haemodynamic improvements in $\mathrm{Cpc}-\mathrm{PH}$ patients after iNO administration [4] according to Gerges et al. [1]. The improved RV afterload with a concomitant $8 \%$ of Rup increase, indicates a decrease of distal PVR $[1,4]$.

Gerges et al. [1] proposed DPG as a surrogate of Rup, which is debatable, since while Rup corresponds to small PAs and arterioles resistances, DPG $(>7 \mathrm{mmHg})$ defines the presence of PVD associated with the increase of pulsatile and/or stationary components of RV afterload.

In conclusion, Gerges et al. [1] have made an "in-depth" effort to link haemodynamics to PVD by partitioning the stationary component of the RV afterload in PH-LHD patients. We can speculate that $Z$ up could provide a simple haemodynamic tool to characterise the different spectrum of RV afterload and the relative contribution of large and small vessel disease in PH states regardless of the PAOP, including the inverse relationship with $\mathrm{HR}$ as an indirect RV functional response. 
Juan C. Grignola ${ }^{1,2}$, Pedro Trujillo ${ }^{2,3}$ and Enric Domingo ${ }^{4,5}$

${ }^{1}$ Dept of Pathophysiology, Hospital de Clínicas, School of Medicine, Universidad de la República, Montevideo, Uruguay. ${ }^{2}$ Pulmonary Hypertension Unit, Hospital Maciel, Ministerio de Salud Pública, Montevideo, Uruguay. ${ }^{3}$ Cardiology Dept, Centro Cardiovascular Universitario, Hospital de Clínicas, School of Medicine, Universidad de la República, Montevideo, Uruguay. ${ }^{4}$ Cardiologia, Hospital Universitari Vall d'Hebron, Barcelona, Spain. ${ }^{5}$ Dept of Physiology, School of Medicine, Universitat Autonoma de Barcelona, Barcelona, Spain.

Correspondence: Juan C. Grignola, Dept of Pathophysiology, Hospital de Clínicas, Facultad de Medicina, Universidad de la República, Avda Italia 2870, PC 11300 Montevideo, Uruguay. E-mail: jgrig@fmed.edu.uy

Received: Oct 042018 | Accepted after revision: Dec 042018

Conflict of interest: None declared.

\section{References}

1 Gerges C, Gerges M, Fesler P, et al. In-depth haemodynamic phenotyping of pulmonary hypertension due to left heart disease. Eur Respir J 2018; 51: 1800067.

2 Fayyaz AU, Edwards WD, Maleszewski JJ, et al. Global pulmonary vascular remodeling in pulmonary hypertension associated with heart failure and preserved or reduced ejection fraction. Circulation 2018; 137: $1796-1810$.

3 Delgado JF, Conde E, Sanchez V, et al. Pulmonary vascular remodeling in pulmonary hypertension due to chronic heart failure. Eur J Heart Fail 2005; 7: 1011-1016.

4 Domingo E, Grignola JC, Trujillo P, et al. Functional and anatomical pulmonary arterial disease in patients with persistent pulmonary hypertension after left valve replacement according to the hemodynamic phenotype. Eur J Heart Fail 2018; 20: Suppl 1, 203.

5 Galiè N, Humbert M, Vachiery J-L. 2015 ESC/ERS Guidelines for the diagnosis and treatment of pulmonary hypertension. Eur Heart J 2015; 37: 67-119.

6 Caravita S, Faini A, Carolino D'Araujo S, et al. Clinical phenotypes and outcomes of pulmonary hypertension due to left heart disease: role of the pre-capillary component. PLoS One 2018; 13: e0199164.

7 Thenappan T, Prins KW, Pritzker MR, et al. The critical role of pulmonary arterial compliance in pulmonary hypertension. Ann Am Thorac Soc 2016; 13: 276-284.

8 Metkus TS, Mullin CJ, Grandin EW, et al. Heart rate dependence of the pulmonary resistance $\times$ compliance $(\mathrm{RC})$ time and impact on right ventricular load. PLoS One 2016; 11: e0166463.

9 Ruiz-Cano MJ, Grignola JC, Cortina J, et al. Composite hemodynamic method of pulsatile and steady right ventricular afterload predicts early outcome after pulmonary endarterectomy for chronic thromboembolic pulmonary hypertension. Int J Cardiol 2012; 158: 475-476.

10 Rommel JJ, Yadav PK, Stouffer GA. Causes and hemodynamic findings in chronic severe pulmonary regurgitation. Catheter Cardiovasc Interv 2018; 92: E197-E203.

11 Grignola JC, Domingo E, Aguilar R, et al. Acute absolute vasodilatation is associated with a lower vascular wal stiffness in pulmonary arterial hypertension. Int J Cardiol 2013; 164: 227-231.

Copyright @ERS 2019

From the authors:

We appreciate the thoughtful comments of J.C. Grignola and colleagues in their letter addressing our article [1]. First, we agree that pulmonary hypertension associated with left heart disease (PH-LHD) is associated with global pulmonary vascular remodelling involving pulmonary arteries, veins and capillaries, as recently reported by FAYYAZ et al. [2]. In fact, we have previously observed increased wall thickness and intimal fibrosis of small pulmonary arteries, even in patients with isolated post-capillary pulmonary hypertension (Ipc- $\mathrm{PH}$ ) or "passive" pulmonary hypertension ( $\mathrm{PH})$ [3]. However, significant pre-capillary pulmonary vascular disease appeared to only be present in patients with combined post and pre-capillary $\mathrm{PH}$ (Cpc-PH), particularly those with elevated diastolic pulmonary vascular pressure gradient (DPG) [3]. Of note, in the study by FAYYAZ et al. [2], diagnosis of PH was solely based on echocardiography. Furthermore, in their study, DPG was $3 \mathrm{mmHg}$ and pulmonary vascular resistance (PVR) was 3.9 WU in

@ERSpublications

Haemodynamic estimates of pulmonary vascular disease are a major requirement for the understanding and management of patients with pulmonary hypertension due to left heart disease http://ow.ly/ABsl30nuXX2

Cite this article as: Gerges C, Gerges M, Lang IM, et al. Pulmonary hypertension associated with left heart disease: efforts to improve the meaning of haemodynamic phenotypes. Eur Respir J 2019; 53: 1802393 [https://doi.org/10.1183/13993003.02393-2018]. 
the subgroup of 30 patients with available invasive haemodynamic data, thus resembling the haemodynamic phenotype of Ipc-PH. Application of these data are therefore limited to Ipc-PH and cannot be applied to Cpc-PH.

Our recent manuscript in the European Respiratory Journal demonstrates that in Cpc- $\mathrm{PH}$ effective capillary pressure $\left(P_{\mathrm{c}^{\prime}}\right)$, which reflects the pressure in pre-capillary small pulmonary arteries and pulmonary capillaries, is significantly higher than mean pulmonary arterial wedge pressure (mPAWP), resulting in a decrease in upstream resistance ( $R$ up) [1]. In contrast, $P_{c^{\prime}}$ and $\mathrm{mPAWP}$ are at the same level in Ipc- $\mathrm{PH}$, leading to a high $\mathrm{Rup}$, close to $100 \%$. These results are in line with our previous observations from histomorphometric analyses of lung samples from PH-LHD patients, where arteriolar changes were most pronounced in patients with $\mathrm{Cpc}-\mathrm{PH}$ [3]. Rup may therefore be used to provide haemodynamic proof of pre-capillary pulmonary vascular disease. However, this needs to be tested in further studies.

J.C. Grignola and colleagues propose upstream impedance ( $Z$ up), a composite haemodynamic index, which is thought to assess pulsatile and steady components of right ventricular (RV) afterload simultaneously. The clinical metric most often used to assess RV afterload is PVR. However, even when measured from multiple points derived from pressure-flow curves during exercise, PVR provides only information on peripheral arterial function, but not on proximal arterial function, and gives an incomplete description of all the forces that oppose RV flow output [4]. Pulmonary vascular impedance spectra (PVZ) capture the impact of proximal and peripheral arterial structure and function on RV function. PVZ is a complex, frequency-dependent function of pulsatile pulmonary pressure and flow that is sensitive to changes in proximal, intermediate and peripheral pulmonary arterial resistance and compliance. Typically, Fourier series analyses are performed to calculate PVZ from pressure and flow waves measured at a single location in the pulmonary circulation. J.C. Grignola and colleagues propose that $Z$ up may be a simple alternative to complex and cumbersome PVZ calculations potentially applicable in clinical routine. However, there is a lack of studies that have confirmed that $Z_{u p}$ is indeed related to impedance of the pulmonary circulation via simultaneous assessment. Furthermore, it has not been demonstrated that $Z$ up truly correlates with the pulmonary vascular changes to which J.C. Grignola and colleagues refer.

Interestingly, the proposed formula for the calculation of $Z$ up is strikingly similar to the $R$ up formula:

$$
\begin{aligned}
\text { Zup } & =\frac{\mathrm{mPAP}-\mathrm{dPAP}}{\mathrm{mPAP}-\mathrm{mPAWP}} \\
R_{u p} & =\frac{\mathrm{mPAP}-P c^{\prime}}{\mathrm{mPAP}-\mathrm{mPAWP}}
\end{aligned}
$$

where $\mathrm{mPAP}$ is mean pulmonary artery pressure and $\mathrm{dPAP}$ is diastolic pulmonary artery pressure.

As can be seen above, the only difference between the two formulae is that $P_{c^{\prime}}$ is substituted by dPAP. Indeed, as already hypothesised by Chemla et al. [5], $P_{c^{\prime}}$ is within the limits of dPAP and mPAWP. In healthy subjects or patients with Ipc-PH, where the dPAP-mPAWP gradient is small $[1], P_{c^{\prime}}$ may be theoretically substituted by dPAP. However, pre-capillary pulmonary vascular disease may alter the validity of this assumption, with $\mathrm{dPAP}$ and $P_{\mathrm{c}^{\prime}}$ being significantly above mPAWP [1]. We found that in patients with idiopathic pulmonary arterial hypertension, estimation of $P_{\mathrm{c}^{\prime}}$ using $\mathrm{dPAP}$ lacked precision and accuracy (bias $8.7 \mathrm{mmHg}$, limits of agreement from -4.3 to $21,7 \mathrm{mmHg}$ ). Hence, despite similar formulae, Zup cannot replace Rup for the assessment of upstream and downstream resistance.

Christian Gerges, Mario Gerges and Irene M. Lang

Dept of Internal Medicine II, Division of Cardiology, Vienna General Hospital, Medical University of Vienna, Vienna, Austria.

Correspondence: Irene M. Lang, Dept of Internal Medicine II, Division of Cardiology, Medical University of Vienna, Währinger Gürtel 18-20, 1090 Vienna, Austria. E-mail: irene.lang@meduniwien.ac.at

Received: Dec 172018 | Accepted after revision: Dec 182018

Conflict of interest: C. Gerges reports receiving the following during the conduct of the study: grants from United Therapeutics Corporation, Bayer Healthcare and Actelion Pharmaceuticals, and personal fees from GlaxoSmithKline, AOPOrphan and Actelion. M. Gerges reports receiving the following during the conduct of the study: grants from United Therapeutics Corporation, Bayer Healthcare and Actelion Pharmaceuticals. M. Gerges reports receiving the following outside the submitted work: personal fees from GlaxoSmithKline, AOPOrphan and Actelion. I.M. Lang reports receiving the following during the conduct of the study: grants from United Therapeutics Corporation, Bayer AG and Actelion Pharmaceuticals. I.M. Lang reports receiving the following outside the submitted work: grants, personal fees and non-financial support from Actelion, AOPOrphan Pharmaceuticals, and United Therapeutics Corporation; and other support from AstraZeneca, Servier, Medtronic and Novartis. 
Support statement: This research was funded by educational grants from Actelion Pharmaceuticals Ltd (00283GMS\&C), Bayer HealthCare Pharmaceuticals (grant number 15662 and IIR 18495), SFB-54 and United Therapeutics Corporation (grant number REG-NC-002).

\section{References}

1 Gerges C, Gerges M, Fesler P, et al. In-depth haemodynamic phenotyping of pulmonary hypertension due to left heart disease. Eur Respir J 2018; 51: 1800067.

2 Fayyaz AU, Edwards WD, Maleszewski JJ, et al. global pulmonary vascular remodeling in pulmonary hypertension associated with heart failure and preserved or reduced ejection fraction. Circulation 2018; 137: 1796-1810.

3 Gerges C, Gerges M, Lang MB, et al. Diastolic pulmonary vascular pressure gradient: a predictor of prognosis in "out-of-proportion" pulmonary hypertension. Chest 2013; 143: 758-766.

4 Chesler NC, Roldan A, Vanderpool RR, et al. How to measure pulmonary vascular and right ventricular function. Conf Proc IEEE Eng Med Biol Soc 2009; 2009: 177-180.

5 Chemla D, Lau EM, Papelier Y, et al. Pulmonary vascular resistance and compliance relationship in pulmonary hypertension. Eur Respir J 2015; 46: 1178-1189. 